I. Bei der Aktivität der Enzyme CK, G6P-DH und ALD konnte keine Differenzierung zwischen den Selektionslinien festgestellt werden.

2. Die Enzyme LDH und LDHI zeigten bei den auf hohe Belastbarkeit selektierten Tieren eine signifikant geringere Aktivität.

3. Das Enzym AP zeigte bei den auf hohe Belastbarkeit selektierten Tieren eine signifikant höhere Aktivität.

Die Frage nach dem direkten physiologischen Zusammenhang zwischen den im Blutserum gemessenen Enzymaktivitäten und der Ausprägung des Merkmals Belastbarkeit muß weiteren Untersuchungen vorbehalten bleiben.

\title{
INTERAKTIONEN ZWISCHEN MASTMETHODEN (KÄLBERAST-JUNGSTIERMAST) UND Rassen bei MitTeleuropaischen RiNDERN
}

\section{A. El-Hakim, H. Eichinger und F. Pirchner.-Lehrstuhl für Tierzucht Technische Universität} München 8050 Freising, Weihenstephan.

Kälber von fünf Rassen (Braunvieh, Fleckvieh, Grauvieh, Pinzgauer, Schwarzbunt) wurden zum Teil als Milchmastkälber, zum Teil als Jährlingsstiere geschlachtet. Im ersten Fall wurden die Tiere gleichem Alter (3 $1 / 2$ Monate), im letzteren bei ungefähr vergleichbarem Ausmastgrad geschlachtet. Unterschiede zwischen Rassen waren fast überall hoch signifikant, während die Interaktion Rasse $\times$ Mastmethode bei p. Ioo Brust, p. roo Kotelett, p. Ioo Keule, sowie bei Schlachtkörpergwicht/Alter statistische Signifikanz erreichte. Wenn bei vergleichbarem Ausmastgrad geschlachtet wird, sind Unterschiede zwischen Rassen im Hinblick auf Nettozunahme relativ gering und kleine Rassen gegenüber großen Rassen nicht benachteiligt. Bei den Schlachtqualitätsmerkmalen waren die drei Rassen Braunvieh, Fleckvieh, Grauvieh gegenüber Pinzgauer und insbesondere gegenüber Schwarzbunt, die im wesentlichen Schwarzbunt-Holsteinkreuzungen darstellten, günstiger gelegen.

Es kann gefolgert werden, daß bei Eigenschaften, wo keine Interaktionen zwischen Rasse und Mastmethoden aufgetreten sind, auch keine innerhalb von Rassen zu erwarten sind. Bei Eigenschaften, wo sich Interaktionen gezeigt haben, wäre noch zu prüfen, ob die kleineren genetischen Unterschiede innerhalb von Rassen zu Interaktionen führen.

\section{Untersuchungen üBer den Einfluss des maternalen EfFektes auf die Heritabilität der Wurfgrösse bei Sauen}

\author{
Ingrid Alsing. - Lehrstuhl für Tieräucht Technische Universität München D-8050 Freising- \\ Weihenstephan.
}

Anhand von Landrassesauen des Niederbayrischen Schweinezuchtverbandes wurden Untersuchungen über eine mögliche negative Korrelation zwischen direkt genetischen und maternalen Effekten durchgeführt, die in einer verzögerten physiologischen Reife der Nachkommen aus großen Würfen, durch ungünstige Umwelt relativ zu Nachkommen aus kleineren Würfen begründet. Zur Heritabilitätsschätzung wurden gewichtete Töchter-Mütter-Regressionen für die Merkmale lebend und insgesamt geborene Ferkel berechnet, und zwar vom jeweils ersten Wurf der Töchter sowohl auf ihre direkten Abstammungswürfe als auch auf andere Würfe ihrer Mütter. Aus den unterschiedlichen Heritabilitätswerten für den direkten und den indirekten TochterMutter-Wurf-Vergleich läßt sich die Tendenz maternaler Effekte erkennen, ausgedrückt in den niedrigeren Regressionskoeffizienten beider Merkmale aus der direkten Schätzmethode. Nach Unterteilung der Abstammungswürfe der Töchter in Paritäten zeigt sich, daß der maternale Effekt dort am stärksten wirksam wird, wo Tochter und Mutter ihren ersten Wurf brachten. Dieser negative Umwelteinfluß witd möglicherweise mit steigender Wurfzahl der Mutter von einem positiven genetischen Einfluß überdeckt. Bei einer Aufteilung jeder Paritätengruppe nach Herkunftswurfgrößen werden die Werte der Regressionen sowhol für sehr große als auch kleine Herkunftswürfe wie erwartet negativ. Diese Ergebnisse unterstützen die Hypothese, da $\beta$ auf kleine Würfe große folgen und umgekehrt und die Heritabilitätswerte für unterschiedliche Wurfgrößen unterschiedlich sind. 